\title{
Research on the X-ray wavelength division multiplexing technology for blackout region communication
}

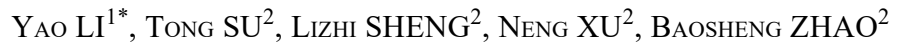 \\ ${ }^{1}$ Shaanxi Province Key Laboratory of Thin Films Technology and Optical Test, \\ College of Optoelectronic Engineering, Xi'an Technological University, \\ Xi' an 710021, China \\ ${ }^{2}$ State Key Laboratory of Transient Optics and Photonics, \\ Xi'an Institute of Optics and Precision Mechanics (XIOPM), \\ Chinese Academy of Sciences (CAS), Xi' an 710119, China \\ *Corresponding author: liyao_xatu@163.com
}

\begin{abstract}
After the concept of X-ray communication was proposed, its application in complex electromagnetic environment has received more attention, such as data transmission in re-enter special electromagnetic condition. In this article, a new type of X-ray source was introduced firstly, which was expected to generate multiple characteristic lines and achieve wavelength division multiplexing technology in X-ray band. Then an experimental platform was built for analyzing transmission characteristics of X-ray photon in various plasma media. Finally, the calculation model for a link power equation was given. Experiment results show that transmittance of 8-18 keV X-ray signal is relatively stable, atomic numbers from 29 to 42 are the most suitable materials for wavelength division multiplexing, the X-ray communication system is expected to realize about $200 \mathrm{kbps}$ data transmission rate in adjacent space.
\end{abstract}

Keywords: X-ray communication, plasma sheath, wavelength division multiplexing.

\section{Introduction}

In 2007, Keith Gendreau, a physicist from NASA's Goddard Space Flight Center, introduced XCOM (XCOM, X-ray communication) for the first time [1] . XCOM method uses $\mathrm{X}$-ray photons as carriers to realize information transmission. Thus, XCOM has the following merits [2]: firstly, X-ray carrier has a higher photon energy, usually on the orders of $\mathrm{keV}$. Therefore, it is expected to be used in the field of deep space communication. Secondly, based on the high frequency and low divergence angle characteristics, XCOM is expected to transmit large amounts of data in a short time and reduce the risk of eavesdropping. Finally, owing to the strong penetrating power of X-ray photon, XCOM can be used for some special fields, such as data transmission through 
a steel plate shield or plasma sheath environment. Many scholars have conducted related research in the field of XCOM since the concept was introduced. In 2015, Dr. Keith Gendreau has verified the concept of XCOM by using an ultraviolet modulated X-ray source [ $\underline{3}$ ]. In 2012-2015, ZHAO et al. established a XCOM demonstration system in a $10 \mathrm{~m}$ vacuum pipe based on Wolter-IX-ray focusing optics and X-ray detector. Their system can achieve about Mbps transmitting speed [4]. In 2017, Li et al. introduced a XCOM method on the basis of a laser modulated X-ray source [ $\underline{5}$ ]. However, their scheme has no relevant experimental data [ $[\underline{6}, \underline{6}]$. In 2018, Li et al. has analyzed the transmittance of $X$-ray signals in a static plasma medium for the first time [7]. Experiments results indicate that $\mathrm{X}$-ray signals can transmit information under the plasma medium, but the number of photons emitted by a digital X-ray source limits the communication index [ $[\underline{7}, \underline{8}$. In 2019, NASA has launched a modulated source for spatial XCOM, planning to achieve a $50 \mathrm{~m}$ XCOM experiment verification on ISS (ISS, International Space Station) in the next few years [9]. However, throughout the development trend of $\mathrm{XCOM}$ technology, increasing the number of emitted X-ray photons is a key factor in improving the core communication parameters (distance, speed and bit-error ratio).

For the existing X-ray generation and modulation schemes, there are NASA's ultraviolet LED modulation (similar to Li's scheme), Standford's filed emission modulation and Zhao's grid-control X-ray source [1ㅣ]. NASA and Standford's method has experienced a twice energy conversion process from ultraviolet light energy to free electrons and then to emit X-ray photons. Due to the limitation of electron multipliers, the number of emitted photons was limited. As a result, they can only offer $10^{3}-10^{6} \mathrm{cps}$ order of emitted photons.

However, as for the grid-control X-ray source, although it experienced an energy conversion process and the number of emitted electrons is about $10^{14}-10^{15} \mathrm{cps}$ order, the electron-X-ray conversion efficiency is only $10^{-4}-10^{-6}$ and the electron transmittance of the grid part is about $20 \%-30 \%$ [11]. In addition, considering the factors of heat dissipation, the output power of X-ray source cannot be increased indefinitely. For the existing XCOM method, IM/DD (intensity modulation/direct detect) is widely used, the characteristic spectrum of X-ray source cannot be fully utilized and communication parameters are limited.

In this paper, a novel structure of X-ray source is introduced to realize WDM (WDM, wavelength division multiplexing). Then, a glow discharge and alkali metal plasma generator are used to analyze the transmittance of X-ray signals under different characteristic spectrum energy segments. The suitable characteristic lines for information transmission in blackout region were introduced. Finally, in the light of X-ray generation and detection mechanism, a calculation model between X-ray power and communication indicators is proposed and analyzed.

\section{Simulation and analysis}

WDM technology is mature in the field of wireless optical communication, but has not been used in X-ray band. The reason is that the existing X-ray source cannot si- 


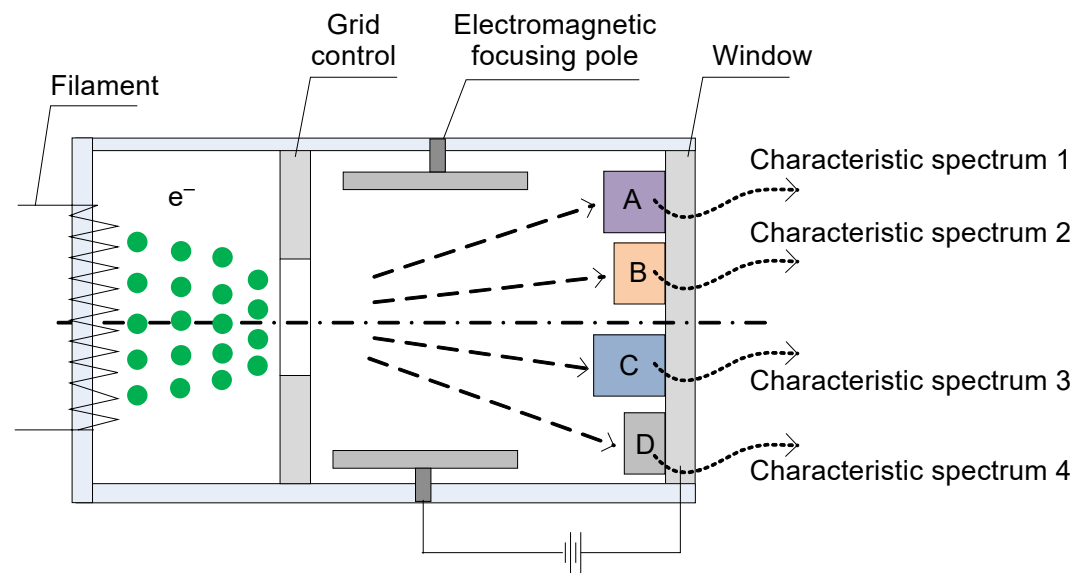

Fig. 1. The structure of WDM X-ray tube.

multaneously generate X-ray signals of multiple energy segments. In order to realize the WDM technology in X-ray band, we designed a multi-anode target X-ray source; the schematic can be seen in Fig. 1.

In this X-ray tube, filament heating first produces free electrons, and then these free electrons are accelerated and controlled by the grid part inside a tube. Finally, by controlling the magnetic focusing pole, the modulated electrons are guided to bombarded different anode targets and next they generate different characteristic lines. However, for this end-window style X-ray source, when the electrons bombard the anode target and generate $\mathrm{X}$-ray photon, part of the emitted $\mathrm{X}$-rays are re-absorbed and the number of emitted photons are restricted [12]. Then the relationship between the emitted X-ray carrier and the anode thickness can be expressed as:

$$
x=\frac{1}{\mu \rho} \ln \frac{I_{\text {in }}}{I_{\text {out }}}
$$

where $\rho$ is the density of the anode, $\mu$ is the mass absorption coefficient (unit $\mathrm{cm}^{2} / \mathrm{g}$ ), $I_{\text {in }}$ and $I_{\text {out }}$ are the intensity of incident and emitted X-ray signals, respectively. When the number of incident electrons is about $3 \times 10^{16} \mathrm{~cm}^{-2}$, the relationship between the emitted X-ray photons $\left(\mathrm{cps} / \mathrm{cm}^{2}\right)$ and the anode target thickness $(\mu \mathrm{m})$ can be seen in Fig. 2.

According to the simulation results of MCNP (MCNP, Monte Carlo $N$-particle transport code) software, the optimal target thickness is generally on the order of micrometer [13]. As the anode voltage increases or atomic number decreases, the optimal target thickness presents an increasing trend. Then the proportion of characteristic spectra under different optimal target thicknesses can be simulated, which is shown in Fig. 3.

When the anode voltage exceeds the $K$ series excitation potential of metal, for lower atomic numbers, the proportion of characteristic spectra is relatively higher. For a higher atomic number, such as molybdenum, tungsten, etc., the specific gravity of continuum 

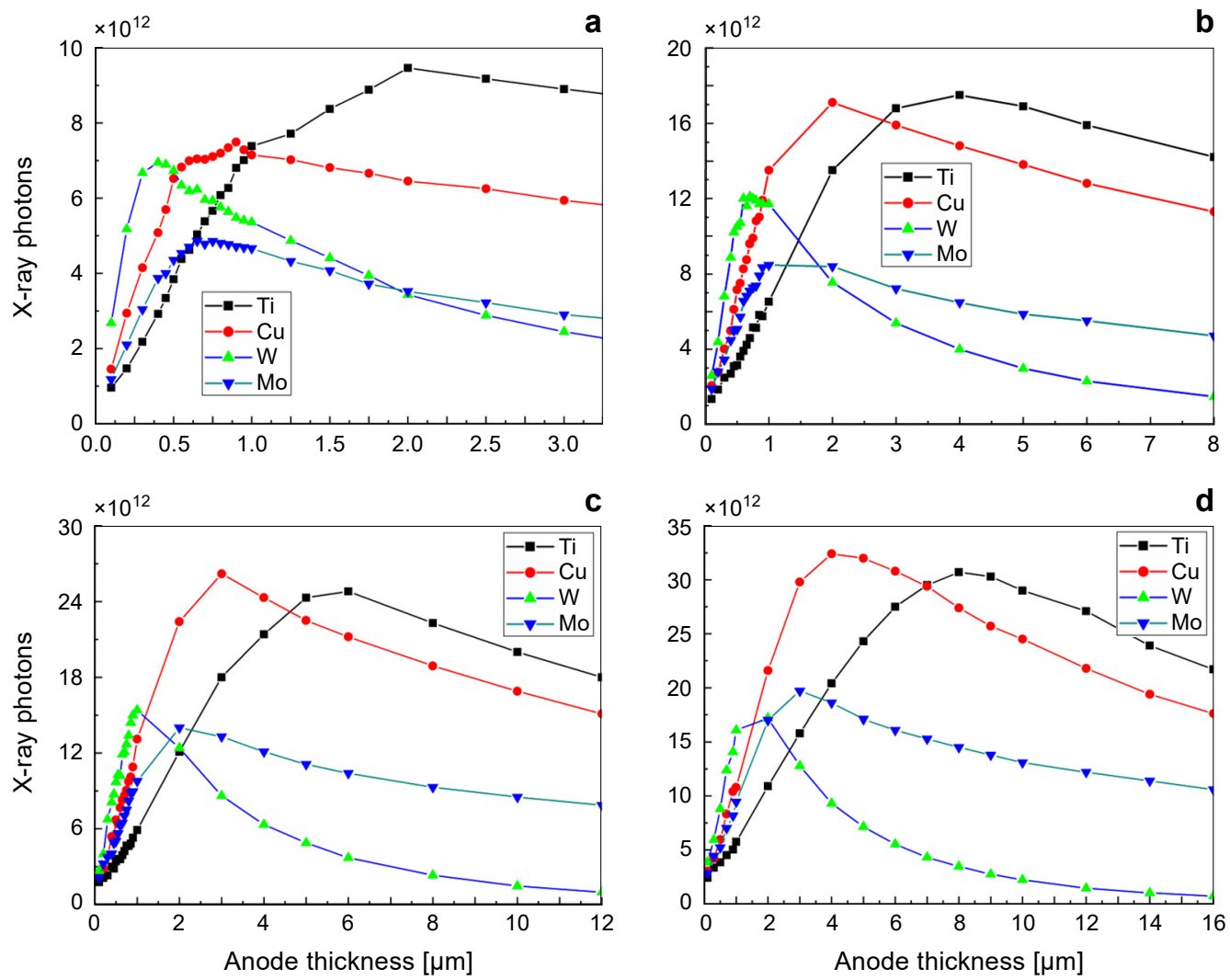

Fig. 2. The relationship between the emitted X-ray photons and the anode target thickness; $20 \mathrm{kV}$ (a), $30 \mathrm{kV}(\mathbf{b}), 40 \mathrm{kV}(\mathbf{c})$, and $50 \mathrm{kV}(\mathbf{d})$ anode voltages.

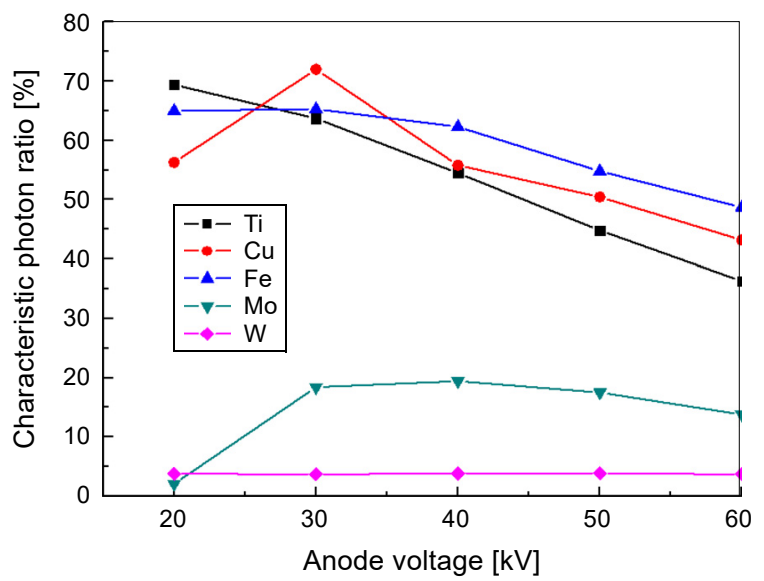

Fig. 3. The proportion of the spectrum for different anode targets. 
spectrum gradually rises. The efficiency of continuum spectrum in a general X-ray tube can be expressed as [14]:

$$
\eta=\alpha Z U
$$

where $\alpha=1.5 \times 10^{-6}, Z$ is the atomic number of the metal anode target, and $U$ represents the anode voltage (unit in $\mathrm{kV}$ ). Considering the application of XCOM in adjacent space condition, the metal anode target with more characteristic spectra and lower atomic number will have a better prospects. Therefore, taking into account the characteristic lines of common metal anode targets, for the general $4-18 \mathrm{keV}$ characteristic spectrum, whether it can penetrate the plasma sheath of the adjacent space becomes a key factor.

\section{Experiment}

It is well known that the transmission model of electromagnetic waves in plasma medium can be regarded as studying the relationship between carrier frequency and plasma frequency. As shown in Eq. (3), the plasma frequency $f_{\mathrm{p}}$ is determined by the electron density $n_{\mathrm{e}}$. If the carrier frequency is much greater than plasma frequency, the transmission co-efficiency of the signal carrier is close to $100 \%[\underline{15}, \underline{16}]$.

$$
f_{\mathrm{p}}=\frac{1}{2 \pi} \sqrt{\frac{n_{\mathrm{e}}(r) e^{2}}{\varepsilon_{0} m_{\mathrm{e}}}} \approx 9 \sqrt{n_{\mathrm{e}}}
$$

However, since the X-ray carrier has a shorter wavelength and strong particle characteristics, it may interact with the particles in plasma sheath and affect the transmission characteristics. Therefore, it is necessary to verify the characteristics of X-ray photon in plasma medium.

In order to study the instantaneous and average transmittance of X-rays carrier in different plasma medium, based on different types of plasma source, the XCOM-plasma demonstration system can be build. Thus, transmission characteristics of X-rays carrier in quasi-uniform and dynamic-dusty plasma medium can be obtained and analyzed, which could provide an experimental basis for the feasibility of XCOM transmission in blackout regions.

\subsection{Quasi-static plasma medium experiment}

For the sake of measuring the transmittance of X-ray signals in quasi-uniform plasma media, a XCOM-plasma experiment system was built based on glow discharge plasma sources [17, 18], which is shown in Fig. 4.

In this plasma source, argon gas was ionized by the ring electrode inside a vacuum chamber and generated a quasi-uniform plasma medium. The electron density of the plasma medium ranges from $6.2 \times 10^{16}-1.23 \times 10^{17} \mathrm{~m}^{-3}$, the length of the plasma medium is $18 \mathrm{~cm}$. 

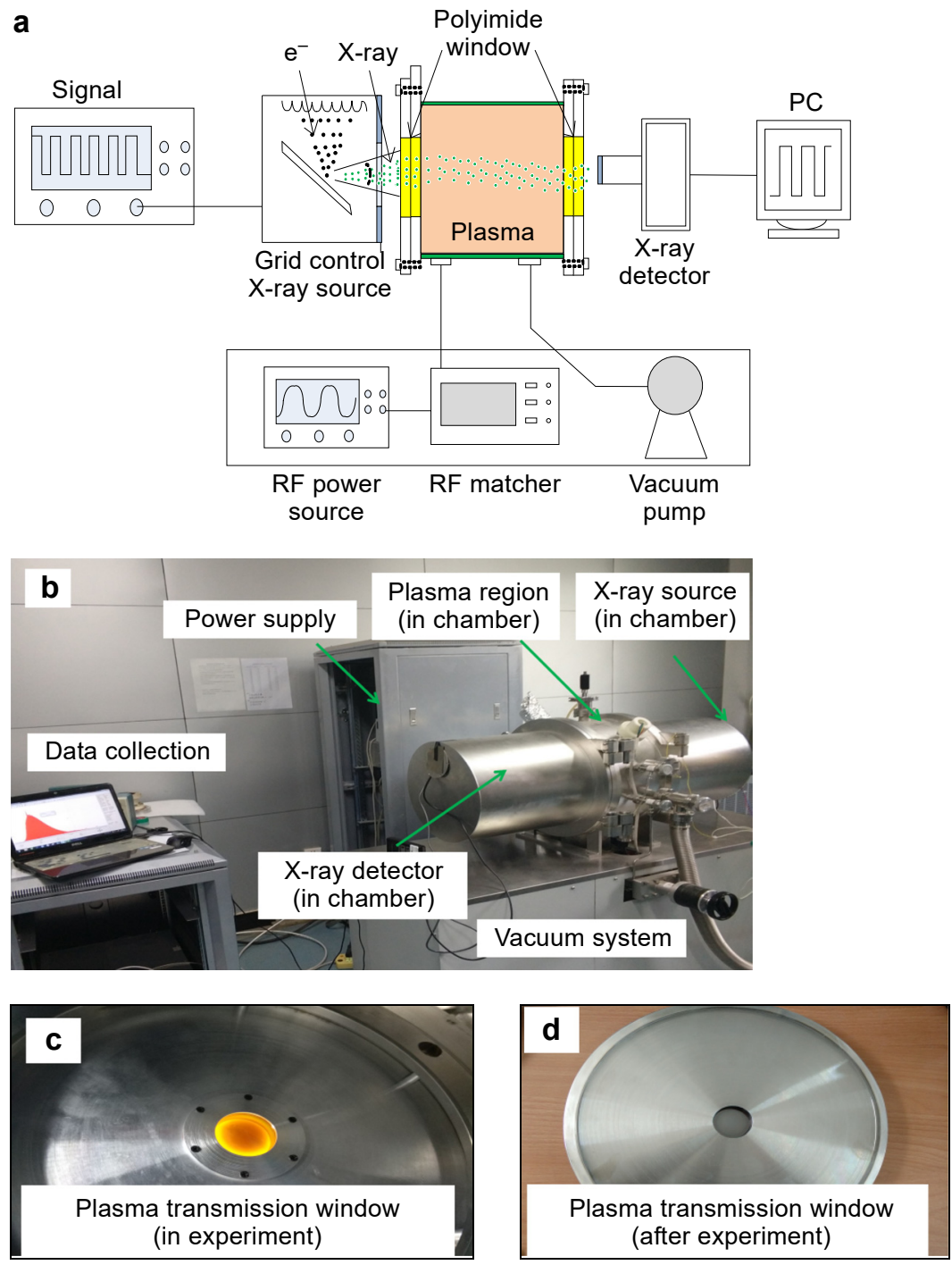

Fig. 4. Schematic (a) and view (b, $, \mathbf{c}, \mathbf{d})$ of the experimental setup of X-ray interact with quasi-static plasma.

According to the experimental results from NASA, the electron density of re-enter plasma medium ranged from $10^{15}$ to $10^{20} \mathrm{~m}^{-3}$. Therefore, this type plasma source can simulate the re-entry condition to a certain extent. In this experiment, a modulated grid-control X-ray tube and SDD (SDD, silicon drift X-ray detector) were fixed at both sides of the plasma chamber. The maximum anode voltage of $\mathrm{X}$-ray tube can reach $25 \mathrm{kV}$. The upper limit of plasma electron density is about $2.5 \times 10^{17} \mathrm{~m}^{-3}$. Then we tested the transmittance of X-ray photons under different characteristic spectrum segments, which can be seen in Fig. 5. 


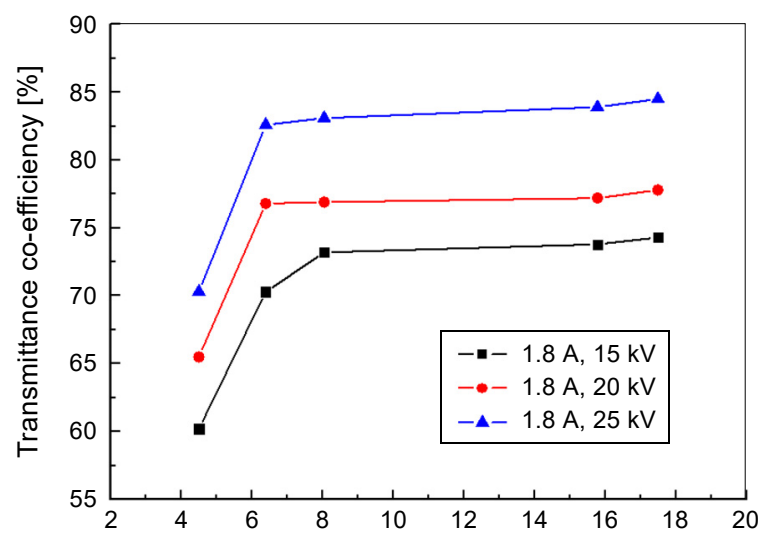

a



Fig. 5. Transmittance of X-ray photon under various energy segments. Electron density $6.2 \times 10^{16} \mathrm{~m}^{-3}$ (a), and transmittance under different plasma electron density (b).

As shown in Fig. 5, when the plasma electron density stays constant, with the increase in anode voltage, the transmittance of X-ray carrier gradually raises. As the carrier energy increases, the transmittance of X-ray photon increases firstly and then remains stable. Moreover, when the electron density of plasma medium rises, the transmittance of X-ray carrier generally decreases. But the transmittance of various electron density presents a constant trend. When the incident carrier energy exceeds $8 \mathrm{keV}$, the transmittance of X-ray signal is basically unchanged under the same plasma parameters. Thus, X-rays carrier can penetrate plasma medium and WDM technique in X-ray band can be realized by using an energy-resolved detector.

\subsection{Dynamic-dusty plasma experiment}

Although the glow discharge plasma source can generate a plasma medium which has the plasma electron density approach blackout condition, this type of plasma medium 
cannot effectively simulate the dynamic, dusty and heat characteristics of the re-enter condition. Thus, an alkali metal ionization plasma source was used for simulating the dynamic and dusty plasma medium [19-21], the advantages are as follows:
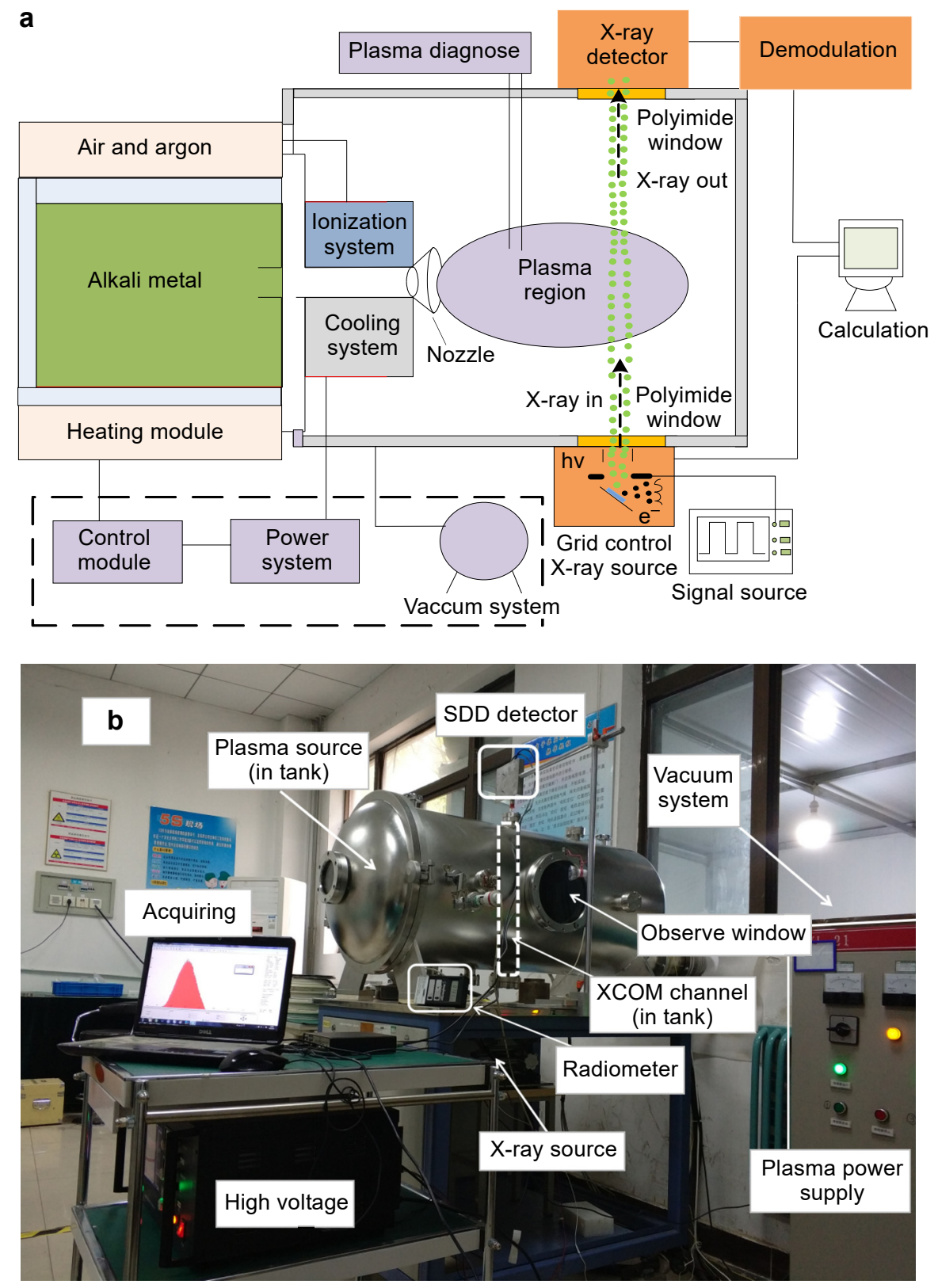

Fig. 6. Schematic and experiment setup of XCOM in dynamic and dusty plasma medium. Schematic (a), experimental site (b), dynamic and dusty plasma jet (c), alkali metal condensed on an observation window (d), and X-ray transmission window (e). 

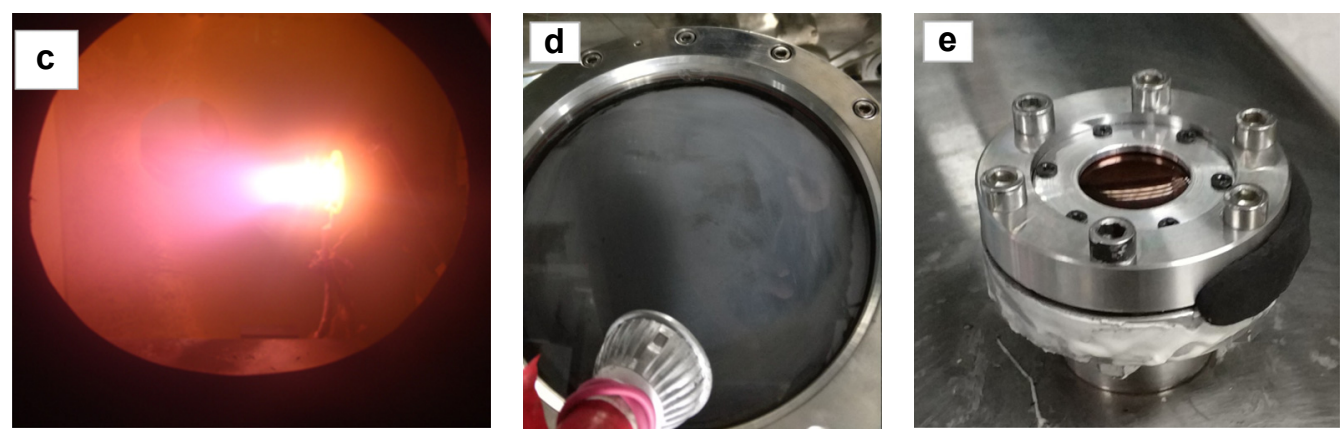

Fig. 6. Continued.

1) The plasma electron density is relatively higher than a general glow discharge plasma source (about $10^{15}-10^{20} \mathrm{~m}^{-3}$ ).

2) The duration time of plasma plume is about $10 \mathrm{~min}$, the plasma temperature at nozzle part is close to $1800 \mathrm{~K}$.

3) The plasma flow generated at the nozzle part has a dynamic, non-uniform and dusty characteristics, which is close to the blackout condition.

In addition, the plasma thickness is about $15-20 \mathrm{~cm}$, the pressure of this chamber is $10^{-4} \mathrm{~Pa}$. Therefore, based on this plasma sources, a XCOM experimental platform was constructed to measure the transmittance under various energy segments, which can be seen in Fig. 6 .

In this experiment, a Langmuir probe was used for detecting the electron density at different times; the results can be seen in Fig. 7.

When the plasma generation conditions stay constant, with the ionization of alkali metal, the electron density of a dusty plasma jet gradually increases and reaches the maximum value. After the reduction of alkali metal materials, the electron density con-

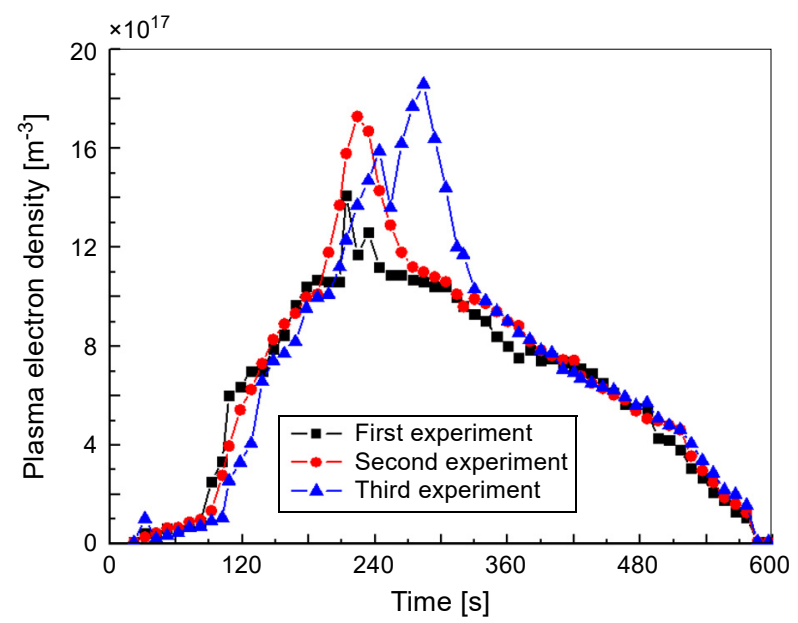

Fig. 7. Plasma electron density at different times. 


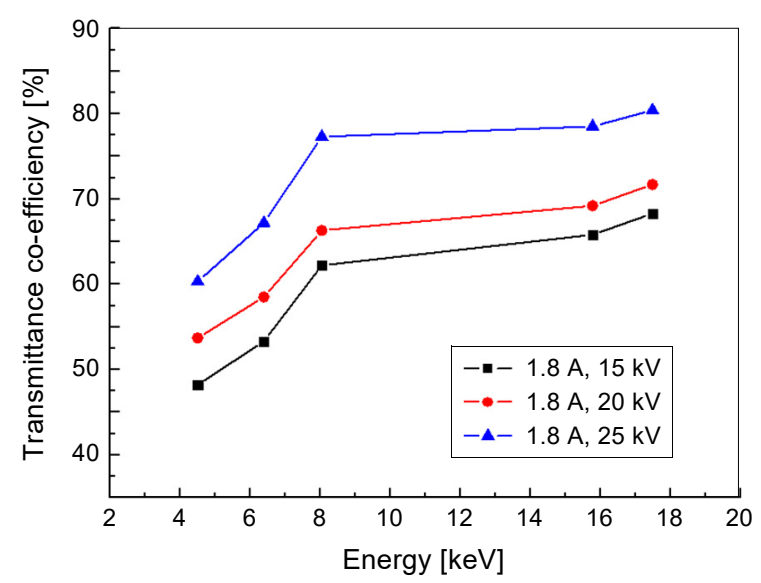

Fig. 8. Transmission co-efficiency of X-ray carrier under various energy segments.

stantly decreases to the normal value. The peak electron density and duration time of this type of a plasma source is $1.85 \times 10^{18} \mathrm{~m}^{-3}$ and $10 \mathrm{~min}$, respectively. Then the average transmission co-efficiency under various energy bands were tested, as shown in Fig. 8.

As shown in Fig. 8, when the photon energy increases, the transmittance co-efficiency first increases and then remains stable, and the average transmittance in dynamic -dusty plasma medium is relatively lower than the glow discharge source. The reasons can be explained as follows $[\underline{22}, \underline{23}]$ :

1) The plasma electron density is relatively higher, and the interactions between $\mathrm{X}$-ray carriers and particles become more frequency.

2) The unionized and partially ionized alkali metal particles will have a certain degree of blocking effect on the emit X-ray signals.

3) The dynamic effects of the plasma will have a certain degree of additional modulation effect on the X-ray signals, which has some influence on the signal transmission.

According to the results from the above experiments, when the photon energy of transmittance signal is greater than $8 \mathrm{keV}$, the transmission co-efficiency tends to be stable. Therefore, taking into account the efficiency of the high voltage module and the existing X-ray detector, the metal material with atomic numbers from 29 to 42 are most suitable for WDM (with characteristics spectrum 8-18 keV). For blackout region communication, it is not necessary to use a hard X-ray carrier, as long as the photon energy is greater than a certain threshold, and the X-ray signal can effectively penetrate the plasma sheath.

\section{Restriction and discussion}

For any communication system, the emit source and detector are important factors limiting communication indexes, and XCOM is no exception. In the detect part, the existing SDD has an energy resolution of $125 \mathrm{eV}$, and the maximum counting rate is about 
5 Mcps, which satisfies the transmission requirement of re-enter condition $[\underline{24}, \underline{25}]$. Because of the X-ray generation mechanism's restriction, the number of emitted photons is a key factor which restricts the improvement of the communication index. Based on a XCOM model, the link power transmission equation is

$$
P_{\mathrm{D}}=\frac{P_{\mathrm{T}} T \eta_{\mathrm{f}} A_{\mathrm{E}} \eta_{\mathrm{d}}}{\pi\left(L \tan \frac{\omega}{2}\right)^{2}}=V E k_{\mathrm{s}}
$$

where $P_{\mathrm{T}}$ represents the output power of X-ray tube, which was limited by the X-ray generation mechanism and anode target thermal load capacity; $T$ represents the transmittance of X-ray signal in re-enter plasma condition; $\eta_{\mathrm{f}}$ and $\eta_{\mathrm{d}}$ are the efficiency of collimation optics and detectors, respectively; $A_{\mathrm{E}}$ is the effective collection area of the $\mathrm{X}$-ray focusing optics; $L$ is the transmittance distance, $\omega$ is the divergence angle of the emit carrier; $V$ is the communication speed, $E$ and $k_{\mathrm{s}}$ are the average photon energy and the number of photons per bit, respectively [7].

In order to analyze the constraints between the XCOM links, set the parameters as follows [다-28]: the emitted X-ray power $P_{\mathrm{T}}=5 \mathrm{~W}$, the average transmittance of $\mathrm{X}$-ray carrier $(\sim 10 \mathrm{keV})$ is $70 \%$. For a general Wolter style X-ray optics, $\eta_{\mathrm{f}}=0.18 \%$, $A_{\mathrm{E}}=5400 \mathrm{~mm}^{2}, \omega=3 \mathrm{mrad}$, the transmittance distance $L=100 \mathrm{~km}$, and the detect efficiency of SDD is about $100 \%$. According to the BER (BER, bit error ratio) calculation model of XCOM system, when the BER order is $10^{-3}, 10^{-4}$ and $10^{-5}, k_{\mathrm{s}}$ should be 5,7 and 9 cps.

As seen from Fig. 9, simulation results indicate that with the reduction in average photon energy and BER order, the transmittance speed of XCOM system can be increased to some extent. When the average photon energy is about $8 \mathrm{keV}, \mathrm{XCOM}$ method is expected to achieve $200 \mathrm{kbps}$ data transmission in adjacent space. In addition, if the emit power of X-ray source keeps constant, the $k_{\alpha}$ line of $\mathrm{Cu}(8.05 \mathrm{keV})$ can be selected as the main communication band, and $\mathrm{Zn}(8.54 \mathrm{keV}), \mathrm{Zr}(15.77 \mathrm{keV}), \mathrm{Mo}(17.48 \mathrm{keV})$

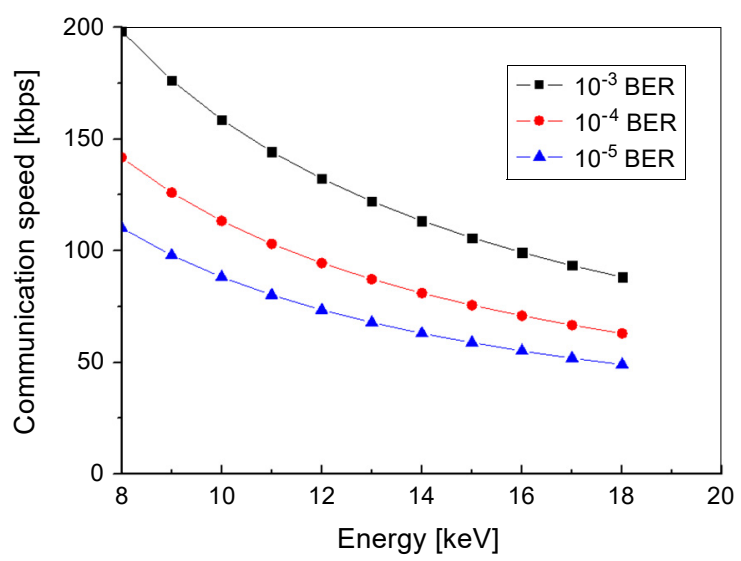

Fig. 9. Potential communication speed under various X-ray energies. 
can be assisted for WDM. Thus, a WDM method can be used for transmitting data on re-enter condition and reducing the requirements for X-ray source to a certain extent.

\section{Conclusion}

As a special wireless communication method, XCOM could achieve an effective data transmission re-enter plasma condition. This paper aims at studying WDM technology in X-ray band. Firstly, an X-ray source that can implement the WDM was proposed. Then the feasibility of scheme and proportions of the spectrum are analyzed. Monte Carol simulation results indicate that lower atomic number metal targets can obtain a higher proportion of the spectrum. Then the transmission co-efficiency of different band carriers in quasi-static plasma and dynamic plasma were tested based on two different types of plasma sources. Experiment results indicate that when the photon energy of a transmittance signal is greater than $8 \mathrm{keV}$, the transmission co-efficiency tends stable. Finally, we analyzed the potential communication speed and the suitable anode materials of the XCOM system.

Acknowledgements - This article was supported by NSFC of China (No. 61901470, No. 62001364 and No. 11805150) and the Basic Research Program of Shaanxi Province Natural Science Foundation (No. 2020JQ-818), the Natural Science Foundation of Shaanxi Provincial Department of Education (No. 20JK0665), and the Collaborative Innovation Center Project of Shaanxi Provincial Department of Education (20JY031). The quasi-static and dynamic-dusty experiments were carried out in the College of Aerospace Science and Technology, XIDIAN University and XIOPM, respectively.

\section{References}

[1] Gendreau K., Next-generation communications: 'Demonstrating the world's first X-ray communication system, FS-2007-10-103-GSFC (TT\#7).; https://gsfctechnology.gsfc.nasa.gov/TechSheets/ XRAY_Goddard_Final.pdf (accessed September 30, 2019).

[2] Song S.B., Xu L.P., Zhang H., BAI Y., Novel X-ray communication based XNAV augmentation method using X-ray detectors, Sensors 15(9), 2015, pp. 22325-22342, DOI: 10.3390/s150922325.

[3] NASA Technology Roadmaps, Communication, navigation, and orbital debris tracking and characterization systems, Report No. TA5 (2015), pp. 12-13; https://www.nasa.gov/sites/default/files/ atoms/files/2015_nasa_technology_roadmaps.pdf (accessed September 30, 2019).

[4] Wang L.Q., Su T., Zhä B.S., Sheng L.Z., Liu Y.A., Liu D., Bit error rate analysis of X-ray communication system, Acta Physica Sinica 64(12), 2015, article 120701, DOI: 10.7498/aps.64.120701.

[5] Li H., TAng X., Hang S., Liu Y., Chen D., Potential application of X-ray communication through a plasma sheath encountered during spacecraft reentry into earth's atmosphere, Journal of Applied Physics 121(12), 2017, article 123101, DOI: 10.1063/1.4978758.

[6] HAng S., Liu Y., Li H., TANG X., Chen D., Temporal characteristic analysis oflaser-modulated pulsed $X$-ray source for space X-ray communication, Nuclear Instruments and Methods in Physics Research Section A: Accelerators, Spectrometers, Detectors and Associated Equipment 887, 2018, pp. 18-26, DOI: 10.1016/j.nima.2018.01.031.

[7] Li Y., Su T., Shi F., Sheng L., Qiang P., Zhao B., Bit error rate analysis of the spatial X-ray communication system, Infrared and Laser Engineering 47(6), 2018, article 622001, DOI: 10.3788/IRLA201847. 0622001. 
[8] Li Y., Su T., Lei F., Xu N., Sheng L.Z., Zhao B.S., X-ray transmission characteristics and potential communication application in plasma region, Acta Physica Sinica 68(4), 2019, article 040401, DOI: 10.7498/aps.68.20181973.

[9] NASA, NASA Set to Demonstration X-ray Communication in Space, https://www.nasa.gov/sites/default/files/atoms/files/winter_2019_final_web_version.pdf (accessed February 2, 2019).

[10] Su T., Tang L., Li Y., Sheng L., Zhao B., An X-ray frequency modulation method and its application in X-ray communication, Optik 199, 2019, article 163263, DOI: $\underline{10.1016 / j . i j l e o .2019 .163263 .}$

[11] Plewes D.B., Vogelstein E., Grid controlled X-ray tube switching time: implications for rapid exposure control, Medical Physics 11(5), 1984, pp. 693-696, DOI: 10.1118/1.595560.

[12] Yue G.Z., Qiu Q., Gao B., Cheng Y., Zhang J., Shimoda H., Chang S., Lu J.P., Zhou O., Generation of continuous and pulsed diagnostic imaging $X$-ray radiation using a carbon-nanotube-based field -emission cathode, Applied Physics Letters 81(2), 2002, pp. 355-357, DOI: 10.1063/1.1492305.

[13] Johnston E.M., Byun S.H., Farquharson M.J., Determination of optimal metallic secondary target thickness, collimation, and exposure parameters for X-ray tube-based polarized EDXRF, X-Ray Spectrometry 46(2), 2017, pp. 93-101, DOI: 10.1002/xrs.2736.

[14] Zschornack G.H., Handbook of X-Ray Data, Springer-Verlag, Berlin, Heidelberg, 2007, DOI: 10.1007/978-3-540-28619-6.

[15] Chen W., Yang L.X., Huang Z.X., Guo L., Propagation characteristics of THz waves in space-time inhomogeneous and fully ionized dusty plasma sheath, Journal of Quantitative Spectroscopy and Radiative Transfer 232, 2019, pp. 66-74, DOI: 10.1016/j.jqsrt.2019.05.001.

[16] Tavant A., Croes V., Lucken R., Lafleur T., Bourdon A., Chabert P., The effects of secondary electron emission on plasma sheath characteristics and electron transport in an $E \times B$ discharge via kinetic simulations, Plasma Sources Science and Technology 27(12), 2018, article 124001, DOI: 10.1088/1361-6595/aaeccd.

[17] YAnG M., Li X.P., XIE K., Liu Y., Liu D., A large volume uniform plasma generator for the experiments of electromagnetic wave propagation in plasma, Physics of Plasmas 20(1), 2013, article 012101, DOI: $10.1063 / 1.4773906$.

[18] Lei F., Li X.P., Liu Y.M., Liu D.L., YANG M., Yu Y.Y., Simulation of a large size inductively coupled plasma generator and comparison with experimental data, AIP Advances 8(1), 2018, artticle 015003, DOI: $10.1063 / 1.5016354$.

[19] Li J.M., Wang Y., Wei J.J., Yuan C.X., Zhou Z.X., Wang X.O., Kudryavtsev A.A., Effects of non-Maxwellian electron distribution function to the propagation coefficients of electromagnetic waves in plasma, IEEE Transactions on Plasma Science 47(1), 2019, pp. 100-103, DOI: 10.1109/ TPS.2018.2883909.

[20] Zhao W., Li Y., Wei X., Xiao L., Zhang B., Li Y., A plasma sheath reproducing method based on adding alkali metal to promote ionization, Journal of Astronautics 39(7), 2018, pp. 115-122.

[21] Chen W., Yang L.X., Huang Z.X., Guo L.X., Research on the propagation characteristics of THz waves in spatial inhomogeneous and time-varying and weakly ionized dusty plasma, IEEE Transactions on Plasma Science 47(10), 2019, pp. 4745-4752, DOI: 10.1109/TPS.2019.2935245.

[22] Jia J.S., Yuan C.X., Gao R.L., Wang Y., Liu Y., Gao J.Y., Zhou Z.X., Sun X.D., Wu J., Li H., Pu S.Z., Propagation of electromagnetic waves in a weakly ionized dusty plasma, Journal of Physics D: Applied Physics 48(46), 2015, article 465201, DOI: 10.1088/0022-3727/48/46/465201.

[23] WANG Z.Y., GuO L.X., DAN L., Li J.T., EM waves propagation characteristics based on modified dielectric constant model in nonuniform weakly ionized dusty plasma, IEEE Transactions on Plasma Science 47(8), 2019, pp. 3978-3985, DOI: 10.1109/TPS.2019.2922195.

[24] Su T., Li Y., Sheng L.Z., Qiang P.F., Chen C., Xu N., Zhao B.S., Space X-ray communication link modeling and power analysis, Acta Photonica Sinca 46(10), 2017, article 1035001, DOI: $10.3788 /$ gZxb20174610.1035001.

[25] Zhang F.R., HAN J.F., RuAn P., Beam pointing analysis and a novel coarse pointing assembly design in space laser communication, Optik 189, 2019, pp. 130-147, DOI: 10.1016/j.ijleo.2019.05.079. 
[26] Xu N., Liu Y.A., Sheng L.Z., Su T., Chen C., Li Y., Zhao B.S., Liu C.L., Novel electronic readout system for micro-channel plate detector with wedge and strip anode, Journal of Modern Optics 66(6), 2019, pp. 697-701, DOI: 10.1080/09500340.2019.1567844.

[27] Liu D., Qiang P.F., Li L.S., Su T., Sheng L.Z., Liu Y.A., Zhao B.S., X-ray focusing optics and its application in X-ray communication system, Acta Physica Sinica 65(1), 2016, article 010703, DOI: 10.7498/aps.65.010703.

[28] Lider V.V., Kirkpatrick-Baez and Wolter X-ray focusing optics (review), Journal of Surface Investigation: X-ray, Synchrotron and Neutron Techniques 13(4), 2019, pp. 670-682, DOI: 10.1134 / $\underline{\text { S102745101904027X. }}$.

Received September 30, 2019 in revised form January 1, 2020 